\title{
Rate Processes on Fractals: Theory, Simulations, and Experiments
}

\author{
Raoul Kopelman ${ }^{1}$
}

Received July 23, 1985

\begin{abstract}
Heterogeneous kinetics are shown to differ drastically from homogeneous kinetics. For the elementary reaction $\mathrm{A}+\mathrm{A} \rightarrow$ products we show that the diffusion-limited reaction rate is proportional to $t^{-h}[\mathrm{~A}]^{2}$ or to $[\mathrm{A}]^{X}$, where $h=1-d_{s} / 2, \quad X=1+2 / d_{s}=(h-2)(h-1)$, and $d_{s}$ is the effective spectral dimension. We note that for $d=d_{s}=1, h=1 / 2$ and $X=3$, for percolating clusters $d_{s}=4 / 3, h=1 / 3$ and $X=5 / 2$, while for "dust" $d_{s}<1,1>h>1 / 2$ and $\infty>X>3$. Scaling arguments, supercomputer simulations and experiments give a consistent picture. The interplay of energetic and geometric heterogeneity results in fractal-like kinetics and is relevant to excitation fusion experiments in porous membranes, films, and polymeric glasses. However, in isotopic mixed crystals, the geometric fractal nature (percolation clusters) dominates.
\end{abstract}

KEY WORDS: Fractal; random walkers; reaction rates; spectral dimension; percolation; energy disorder; porous membranes; excitation fusion.

\section{INTRODUCTION}

A fractal-like kinetics is described here, including scaling arguments, simulations, and experiments on exciton fusion. We believe that these represent a new insight which may be the key to understanding low-dimensional, e.g., heterogeneous transport and chemical kinetics. They also yield a new approach toward the characterization of disordered media via laser excitation and luminescence, with a possible extension toward bioexcitations that range from photosynthetic antenna to nerve cells and the brain. We note that probably more than $90 \%$ of the natural and manmade chemical reactions are heterogeneous, i.e., occur at an interface with an effective dimension lower than 3 (as in a famous cartoon, it is a voyage

\footnotetext{
${ }^{1}$ Department of Chemistry, The University of Michigan, Ann Arbor, Michigan 48109.
} 
in 2.5 dimensions -"Mosaic," January 1985). As examples of heterogeneous reactions we mention industrial catalysis (involving powders, porous materials, etc.), biochemical reactions (membranes, etc.), geochemical (dirt, porous rock), atmospheric (dust, pollutants), electrochemical (rough electrodes) and even interstellar ones (formation of nucleic acids on grains of ice and/or dust). In addition we mention here some heterogeneous photophysical reactions, such as electron-hole recombination on surfaces and in amorphous materials, photoelectron trapping, and, finally, exciton trapping and exciton annihilation (fusion), for which we give experimental evidence. Little is really known on the basis of heterogeneous kinetics except that "frequently the rate-determining step is the actual reaction on the surface."(1) Furthermore, "heterogeneous reactions present a challenge to several branches of science-chemical kinetics, surface, and solid state physics, and surface chemistry; this is a good example of a problem requiring interdisciplinary study and research." (1) We started theoretical, experimental, and simulation studies in this direction over ten years ago. ${ }^{(2)}$ The key realization was that the basic property of interest is the effectively explored space per unit time, the "efficiency" of the random walker, which is the time derivative of the "mean number of distinct sites visited," $\partial S / \partial t$ (the original definition was slightly different, i.e., $S / t$ ). We note here that 19 th century chemical kinetics was similarly defined, i.e., the rate constant $\mathbf{k}$ was proportional to a "reaction volume" per unit time (explicitly, a cylinder whose base is the "reaction cross section" and whose height is the mean free path). ${ }^{(1)}$ Interestingly enough, when Smoluchowski ${ }^{(3)}$ dealt with diffusion-limited kinetics (in three-dimensional homogeneous space) he effectively replaced the "mean free path" by the "diffusion length" of the random walker. It was not possible to replace the Maxwellian cylinder with a "diffusion sphere" as the latter is not linear in

Table I. Diffusion-Limited Elementary Reaction: $A+A \rightarrow$ Products

\begin{tabular}{ccc}
\hline Kinetics & Homogeneous & Heterogeneous (low dim.) $^{a}$ \\
\hline $\begin{array}{c}\text { Transient } \\
\text { (batch) }\end{array}$ & $-\frac{d A}{d t}=K A^{2}$ & $-\frac{d A}{d t}=K_{0} t^{-h} A^{2}, \quad 0 \leqslant h<1$ \\
$\begin{array}{c}\text { Steady state } \\
\text { (steady source) }\end{array}$ & Rate $=K A^{2}$ & Rate $=K_{0} A^{X}, \quad X=\frac{2-h}{1-h}$ \\
\hline
\end{tabular}

${ }^{a}$ Note. For the reaction order $X$ we have $2 \leqslant X<\infty$, while for the heterogeneity exponent $h$ we have $h=1-d_{s} / 2$, where the spectral dimension, $d_{s} \leqslant 2$ for fractals. For simplicity, $C_{\mathrm{A}}$ is designated by $A$. 
time, but rather increases with time as $t^{3 / 2}$. However, the actual exploration volume of the random walker $(S)$ is a fractal object (the Brownian path see Mandelbrot ${ }^{(4)}$ ) whose "mass" (effective volume) only grows as $V^{2 / 3}$, where $V$ is the diffusion sphere volume $\left(4 \pi \bar{r}^{3} / 3\right.$, where $\bar{r}^{2}=D t$ and $D$ is the diffusion constant). Thus the relation of the exploration volume to the "diffusion volume" of the random walker is essentially the same as that of the mass of a Sierpinski web to the embedding volume. ${ }^{(4)}$ It was this exploration volume of a random walker on a percolation cluster that was of interest in our work, ${ }^{(2)}$ as well $s$ in that of de Gennes ${ }^{(5)}$ and other French workers. ${ }^{(6)}$ Is the Brownian path on a fractal a "fractal of a fractal"? What is its effective dimension? And can one generalize from a single random walker to an ensemble of random walkers (as done by Smoluchowski for homogeneous three-dimensional space)? These are the problems of interest

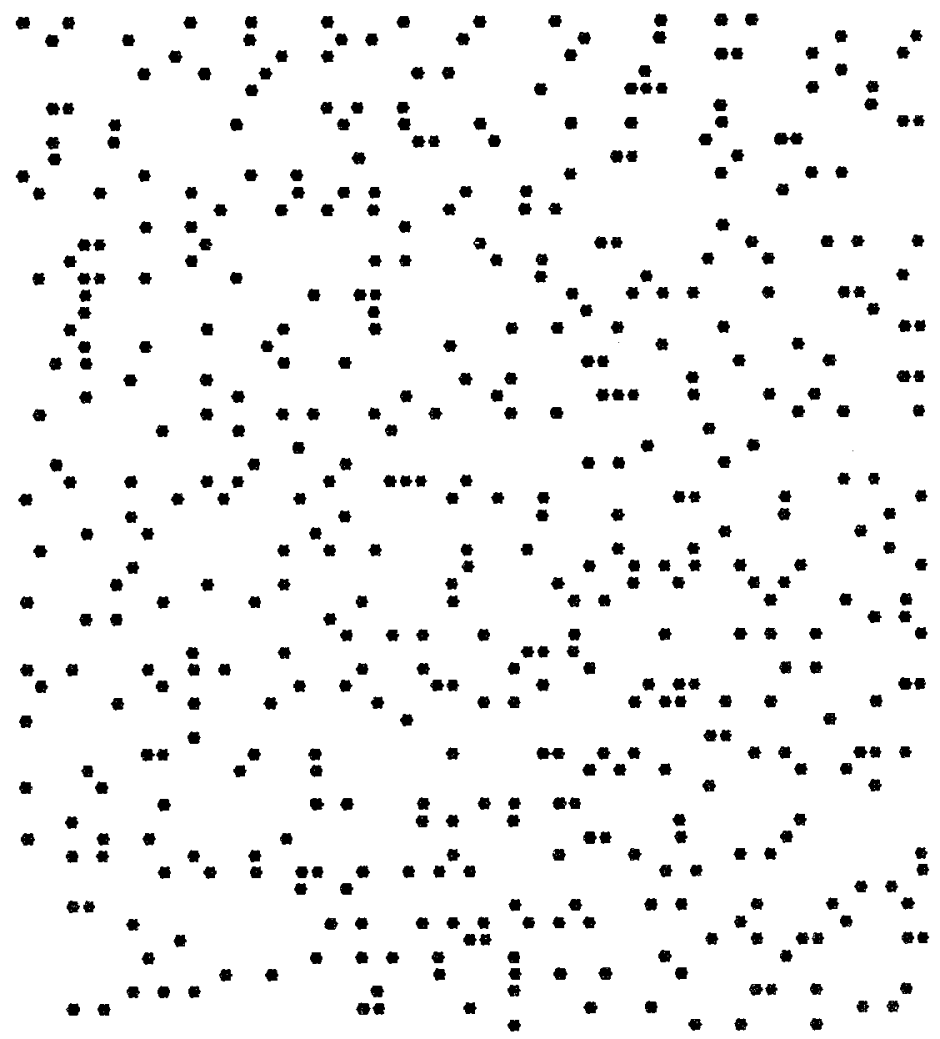

Fig. 1. Schematic simulation of homogeneous kinetics for the elementary reaction $\mathrm{A}+\mathrm{A} \rightarrow$ products. The stars are random walkers on a lattice that fuse or annihilate $(\mathrm{A}+\mathrm{A} \rightarrow \mathrm{A})$ when two of them collide. The resulting rate law is the classical one: $R=K A^{2}$ for both transient and steady-state conditions. 
here, as well as their practical applications. Specifically we report on recent scaling-type theory, simulations and experiments, all of which seem to give a new and consistent picture of diffusion-limited reaction on heterogeneous media. The experiments are on excitations in molecular aggregates in organic alloys, films and membranes. The simulations are on both geometrically and energetically disordered media, with dimensionalities between 1 and 3. The essential message of our results is given in Table I: Figure 1 shows a simulation representing the "Homogeneous" column of Table I, while Fig. 2 represents the "Heterogeneous" column. Figure 3 contains a special case: a schematic picture of effectively one-dimensional pores. The unexpected result is that $X=3$, i.e., an effectively third-order elementary binary reaction.

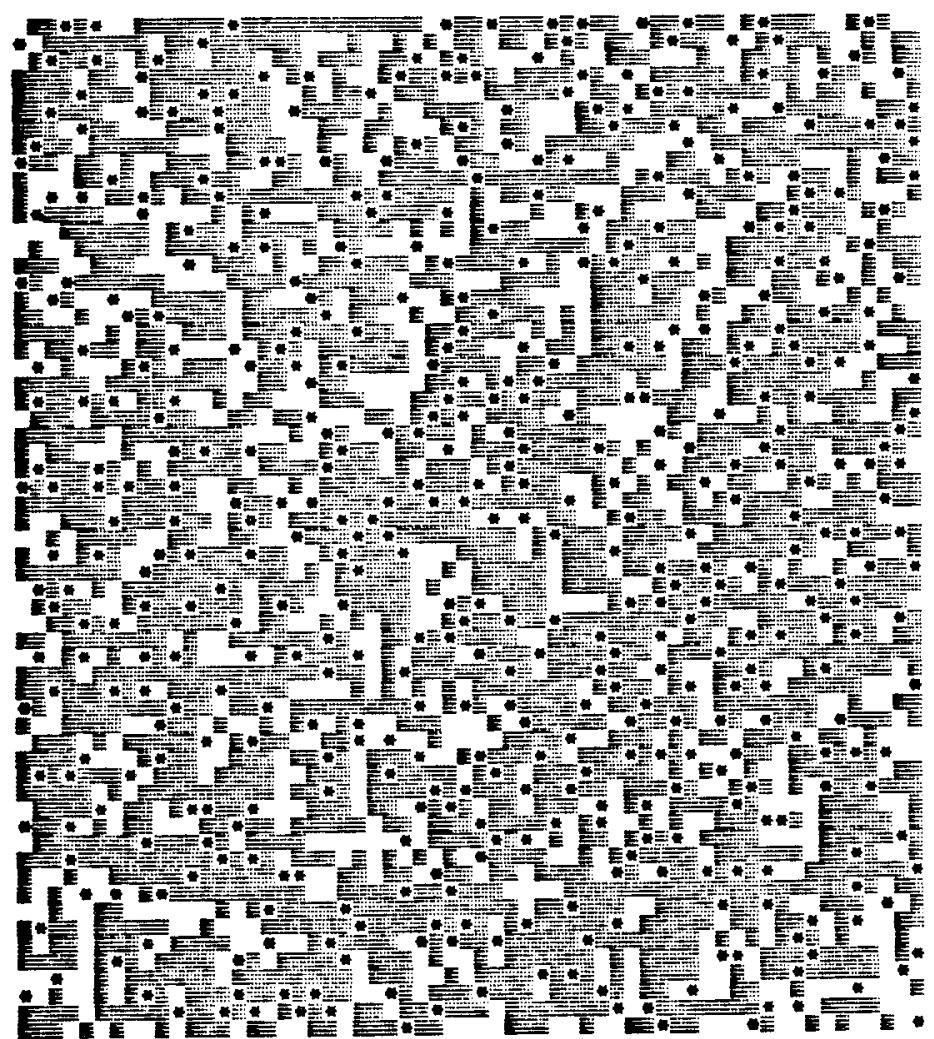

Fig. 2. Schematic simulation of heterogeneous kinetics for the elementary reaction $\mathrm{A}+\mathrm{A} \rightarrow$ products. Same as in Fig. 1 except that the random walkers are constrained on the percolation clusters $(40 \%$ occupation on square lattice). The resulting rate laws are $R=K_{0} t^{-h} A^{2}$ with $h \cong 0.6$ for the transient (batch) reaction and $R=K_{0} A^{X}$ with $X=(h-2) /(h-1) \cong 3.5$ for the steady state (steady source) reaction. 


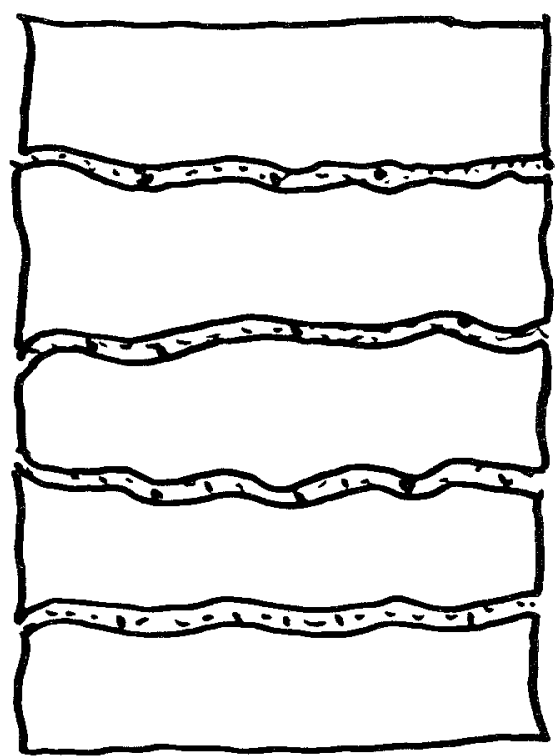

Fig. 3. Schematic representation of reaction in one-dimensional pores of artificial membrane. Note that for the elementary binary reaction $\mathrm{A}+\mathrm{A} \rightarrow$ products one gets $h=1 / 2$ and an effective reaction order $X=3$ (three, not two), provided that the products diffuse through the walls.

\section{THEORY}

The typical reaction of interest is the bimolecular elementary reaction:

$$
\mathrm{A}+\mathrm{B} \rightarrow \text { products }
$$

which has a textbook macroscopic description:

$$
-\frac{d C_{\mathrm{A}}}{d t}=k C_{\mathrm{A}} C_{\mathrm{B}}
$$

where $C(t)$ is the reactant concentration and the "rate constant" $k$ is a constant not only in concentration but also in time. The stochastic approach of Smoluchowski $^{(3)}$ (1917), Chandrasekhar ${ }^{(7)}$ (1943), Noyes ${ }^{(8)}$ (1961), van Kampen ${ }^{(9)}$ (1982), and others (see Calef and Deutsch, ${ }^{(10)} 1983$ ), indeed derives a time independent rate constant $k$ for long times $(t \rightarrow \infty)$, which is proportional to the microscopic diffusion constant $D$ :

$$
k \sim D, \quad t \rightarrow \infty
$$

The standard derivation of Smoluchowski and others starts with the case where the reactant molecules A are random walkers and B are "sitters" 
(traps). This is then extended to the case where both A and B are walkers, via the concept of "relative diffusion" $\left(D_{\mathrm{A}}+D_{\mathrm{B}}\right)$, which is rigorous only for the two-body approximation (one A and one B). The stochastic approach is based on the mean square displacement, which is linear in time for homogeneous systems (and defines D as a proportionality constant). Alternative approaches can be based on the first passage time (the main topic of this symposium) and on the exploration space $S(t)$ ("the mean number of distinct sites visited").

The microscopic quantity, $S(t)$, has been shown to describe the macroscopic rate "constant" (coefficient) $k$, for both homogeneous and locally heterogeneous media. Specifically, it has been shown that

$$
k \sim \frac{d S}{d t}
$$

again giving a simple linear relationship between the macroscopic rate coefficient $k$ and the microscopic quantity $\varepsilon \equiv d S / d t, \varepsilon$ being the "efficiency" of the walker (Argyrakis and Kopelman, ${ }^{(11,12)}$ 1979, 1980; De Gennes, ${ }^{(5)}$ 1983). Equation (4) is strictly true for low concentration $\left(C_{B} \rightarrow 0\right)$ and for a superlattice of B traps, but works well for realistic times and B concentrations (Kopelman, ${ }^{(1)}$ 1976; Klafter et al.. $\left.{ }^{(13)} 1984\right)$.

Scaling conjectures (Klymko and Kopelman, ${ }^{14)}$ 1983; Kang and Redner, ${ }^{(15)}$ 1984) have generalized [Eq. (4)] from the "trapping" reactions to the general reaction, where both $\mathrm{A}$ and $\mathrm{B}$ are random walkers, and, as a special case, to the reaction

$$
2 \mathrm{~A} \rightarrow \text { products }
$$

This reaction has the textbook rate equation,

$$
-\frac{d C_{\mathrm{A}}}{d t}=k C_{\mathrm{A}}^{2}
$$

We note that under a steady state rate $(R)$ of reactant production, one has

$$
R=k C_{\mathrm{A}}^{2}
$$

which is valid only for a time-independent coefficient, $k$.

Does Eq. (4) produce a time-independent rate constant? The answer is yes for a coherent motion of A ( $S \sim t$ and $\varepsilon=$ const) as well as for threedimensional isotropic spaces, where it has been shown (Montroll and Weiss, $\left.{ }^{(16)} 1965\right)$ that $a$ is an asymptotic constant

$$
S=a t, \quad t \rightarrow \infty
$$


so that

$$
k \sim a
$$

However, for locally heterogeneous media, $S=f(t)$. Heterogeneities in the geometry or specific time distributions of hops result in a relationship:

$$
S \sim t^{d_{s} / 2}, \quad d_{s}<2
$$

where $d_{s}$ is the "spectral dimension" (Alexander and Orbach, ${ }^{(17)} 1982$ ), which for fractal objects is a second dimension (in addition to the "fractal" dimension, Mandelbrot, ${ }^{(4)}$ 1983). $d_{s}$ may also be an "effective spectral dimension," for energy disorder that is reflected by an anomalous waiting time distribution (Sherr and Montroll, ${ }^{(18)}$ 1974) or by a specific hopping rate law (Argyrakis, Anacker, and Kopelman, ${ }^{(19)} 1984$ ). From Eq. (10) it is obvious that $\varepsilon=d s / d t$ is no longer constant in time, but

$$
\varepsilon \sim t^{-h}, \quad 0 \leqslant h \leqslant 1
$$

where

$$
\begin{array}{ll}
h=1-d_{s} / 2, & d_{s}<2 \\
h=0, & d_{s}>2
\end{array}
$$

We notice that $(12 \mathrm{~b})$ is only valid for $d=d_{s}=3$, a three-dimensional Euclidean (homogeneous) medium. $d=d_{s}=2$ is a well-known borderline case $(h \approx 0)$. The important result is thus

$$
k \sim t^{-h}, \quad 0 \leqslant h \leqslant 1
$$

where Eqs. (12) still hold.

It can also be shown (Anacker and Kopelman, ${ }^{(20)} 1984$ ) that, for the steady state case, Eq. (7) should be replaced by

$$
R=k_{0} C_{A}^{X}, \quad X \equiv 1+2 / d_{s}=\frac{2-h}{1-h}
$$

giving a new interpretation to the "reaction order" $X$.

Some special cases are the following:

(a) One-dimensional pore $\left(d=d_{s}=1\right)$. Here $h=1 / 2$ and $X=3$ (an unexpected result - see below).

(b) Percolating clusters. Here (Alexander and Orbach, ${ }^{(17)} 1982$ ), for all $d, d_{s} \approx 4 / 3$ and thus $h=1 / 3$ and $X=5 / 2$. The same is true for DLA's (diffusion-limited aggregates, L. Sander, ${ }^{(21)} 1985$ ) and other random fractals (Leyvraz and Stanley, ${ }^{(22)} 1983$ ). 
Table II. Heterogeneity Exponents $(h)$

\begin{tabular}{lcc}
\hline \multicolumn{1}{c}{$h$} & Simulation & Theory \\
\hline Sierpinski gasket (two dimensions) & 0.31 & 0.32 \\
Sierpinski web (three dimensions) & 0.23 & 0.23 \\
Percolation cluster (two dimensions) & 0.33 & 0.34 \\
Percolation cluster (three dimensions) & 0.32 & 0.33 \\
\hline
\end{tabular}

(c) Sierpinski Gasket (Mandelbrot, ${ }^{(4)} 1983$ ): Here $d_{s}=1.36, h=0.32$, and $X=2.45$.

(d) "Dust," which is defined by $0<d_{s}<1$ or $0<h<1 / 2$. Here $3<X<\infty$.

Finally it is important that as soon as $d<3$, one finds that $d_{s} \leqslant 2$. This "empirical" rule is of utmost importance, as it requires replacing equations (8) and (9) by Eqs. (10)-(14), i.e., a fractal-like behavior of the kinetics. We note again that for all fractals $d_{s} \leqslant d_{f}$.

\section{SIMULATIONS}

Most simulations have been performed ${ }^{(20,21)}$ on a cyber 205 "supercomputer." These simulations have been our most direct tool for testing the scaling hypotheses. Here we just summarize the results. The relations $h=1-d_{s} / 2\left(d_{s} \ll 2\right)$, for transient reactions, has been shown to work for (a) percolation clusters, where $d_{s}=4 / 3$, for two- and three-dimensional incipient clusters as well as for long-range incipient percolation clusters; (b) diffusion-limited aggregates (DLA); ${ }^{(21)}$ (c) Sierpinski gaskets (in two and three dimensions), (d) energy disordered, geometrically ordered lattices (Table II). Furthermore, we have tested out the steady state relations, $X=$ $1+2 / d_{s}=(2-h) /(1-h)$. The results are given in Table III. We note that

Table III. Effective Reaction Order $(X)$

\begin{tabular}{lcc}
\hline \multicolumn{1}{c}{$X$} & Simulation $^{a}$ & Theory \\
\hline Cubic (three dimensions) & $X=2.00$ & $X=2.00$ \\
One dimension & $X=3.00$ & $X=3.00$ \\
Sierpinski (two dimensions) & $X=2.45$ & $X=2.46$ \\
Percolation cluster & $X=2.51$ & $X=2.50$ \\
\hline
\end{tabular}

${ }^{a}$ Note. The simulation values are obtained by extrapolation to low density $(A \ll 0)$. 
for the energetically disordered lattices we have modified the "uphill" jump probability by $\exp (-\Delta E / R T)$, where $\Delta E$ is the energy barrier, $T$ the temperature and $R$ the "gas constant." For the geometrically disordered media, the simulated $X$ values are consistent with our analytical (low-density) ${ }^{(20)}$ results to within three significant figures! In addition, we have shown that the energetically disordered lattices do give a fractal-like behavior, i.e., a constant $h$ that does not vary much with time, but is a function of $\Delta E / R T$.

\section{EXPERIMENTS}

Our experiments rely mainly on excitation fusion reactions in disordered media. The heterogeneous medium (i.e., membrane) has an effective dimension of less than 3 , and thus a spectral dimension of less than 2 . The resulting anomalous microscopic diffusion leads to a resulting anomalous macroscopic kinetics. Specifically we study excitation (exciton) reactions, triple + triplet $\rightarrow$ singlet, as both the reactant concentrations and the product (reaction rate) can be monitored continuously in time via the resulting phosphorescence (proportional to triplet population) and the delayed fluorescence (proportional to singlet population, which is proportional to the reaction rate). We study four kinds of fractal-like media.

\subsection{Isotopic Mixed Crystals of Naphthalene}

In these ideal alloys $\left(\mathrm{C}_{10} \mathrm{H}_{8}-\mathrm{C}_{10} \mathrm{D}_{8}\right)$ the random substitutional disorder assures us of getting genuine percolation clusters of naphthalene $\left(\mathrm{C}_{10} \mathrm{H}_{8}\right)$ in naphthalene- $d_{8}\left(\mathrm{C}_{10} \mathrm{D}_{8}\right)$. Energetic considerations (due to zeropoint energy) confine the excitations entirely to the naphthalene clusters ${ }^{(2)}$ (at the experimental temperature of $2 \mathrm{~K}$ ). Thus at the critical percolation concentration $^{(2,4,14,17,19)}$ most of the reactions are confined to incipient percolation clusters, which are well known to be fractals with a spectral dimension of $4 / 3$. This ideal disordered system is the only system where fractal structure is expected down to (but excluding) the molecular level (we note that these alloy crystals are of the highest quality and chemical purity ${ }^{(2,14)}$ ).

For the triplet excitons the effective critical percolation concentration is at about $8 \%$ naphthalene mole fraction (fourth-nearest-neighbor connectivity with a square lattice topology ${ }^{(2)}$ ). In Figure 4 we plot the rate coefficient $k$ vs. time on a $\log \log$ scale. We note that $\left.k \equiv \mid d C_{\mathrm{A}} / d t\right) / C_{\mathrm{A}}^{2} \mid$ and that the phosphorescence $P$ is proportional to $C_{\mathrm{A}}$, while the delayed fluorescence $F$ is proportional to the annihilation rate $\left|d C_{\mathrm{A}} / d t\right|$. For classical kinetics we expect the rate coefficient $k$ to be a constant in time. Indeed, at an alloy concentration $(16 \%)$ well above the critical concen- 


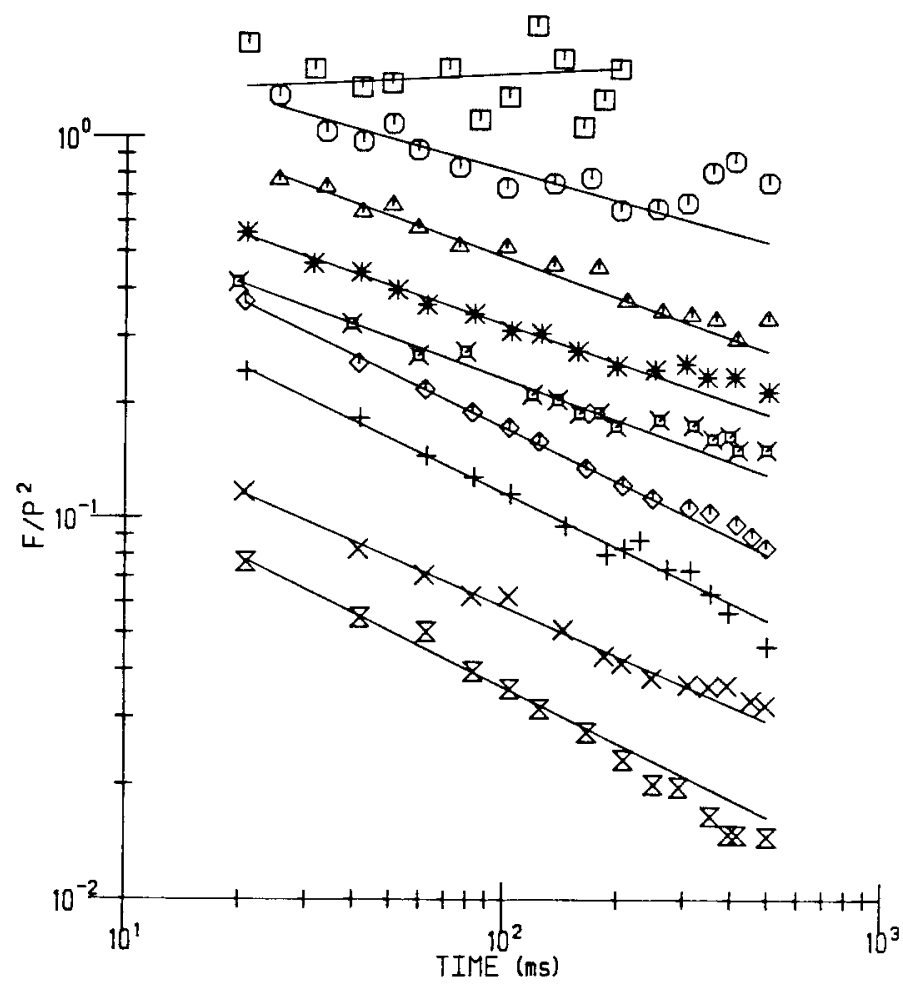

Fig. 4. Rate coefficient $k \equiv F / P^{2}$ vs. time (msec) on a $\log -\log$ plot for triplet exciton fusion (at $2 \mathrm{~K}$ ) on naphthalene- $h_{8}$ in naphthalene- $d_{8}$ perfect crystalline samples. Top to bottom: 16 , $14,12,10,8,6,4,3,2 \mathrm{~mol} \%$. The straight lines are fits to the equation $k \equiv F / P^{2} \sim t^{-h}$ with slope $(-h)$. Curves have been shifted for clarity.

tration $(8 \%)$ we observe such clalssical behavior, i.e., a horizontal line for $k \equiv F / P^{2}$ vs. time (actually on a $\log -\log$ scale, which is equivalent). On the other hand, for the critical alloy concentration $(8 \%)$ we observe the absolute slope $(h)$ to be $0.35 \pm 0.03$, in excellent agreement with the theoretical value: $h=1-d_{s} / 2=1-(4 / 3) / 2=0.33$ (or 0.37 , including corrections due to finite clusters. ${ }^{(5,19)}$ We note that with no free parameters we got an experimental spectral dimension $d_{s}=1.30 \pm 0.06$, i.e., within $3 \%$ of the theory). Above the critical concentration we observe a "fractal to classical crossover with time," i.e., at early times a slope of about 0.35 , crossing over to a zero slope at longer time, as expected. ${ }^{(23,24)}$ On the other hand, below the critical alloy concentration (i.e., at $2 \%-6 \%$ ) we observe the opposite effect of "saturation" with time, as the correlation length approaches the finite cluster size. This is expected ${ }^{(25)}$ for "dusts." These 
transient kinetics data ${ }^{(26,27)}$ are also consistent with anomalous large reaction orders: $X=2.5$ at criticality (see Table III) and $X>10$ at lower alloy concentrations, ${ }^{(27,28)}$ as expected. ${ }^{(20,25)}$

\subsection{Naphthalene Aggregates in Porous Membranes}

Are the domains inside porous polymeric membranes, porous glass and filter papers fractal-like? Many such claims have been made recently, from porous alumina to enzyme surfaces. ${ }^{(29)}$ The fractal dimension of porous vycor (glass) has been claimed ${ }^{(30)}$ to be about 1.7 , even though the exact "fractal nature" of porous glass is under dispute. ${ }^{(31)}$ We show below the fractal-like reaction kinetics of excitation fusion on naphthalene agrregates embedded in such porous materials and membranes. While the interpretation of the data may involve both geometrical and energetical fractal-like aspects, the reaction kinetics is far from the classical picture. Figure 5 shows an $0.2 \mu \mathrm{m}$ porous nylon membrane (Gelman Science, Inc.). Figure 6 shows a $\log k$ vs. $\log t$ plot of the excitation kinetics of naphthalene triplets in such a membrane embedded with naphthalene. Again we see the clear-cut fractal-like behavior, with $h=0.21$. We note that for the classical picture to be valid one requires that $h=0$, that is, a horizontal line of $\ln k$. Similar plots have been obtained for acetate membranes with various pore size distributions, as well as for porous (Vycor) glass ( $h=0.44$ see Fig. 7 ). The effective spectral dimension is thus about $d_{s}^{\text {eff }}=1.1$, which is consistent with the somewhat higher fractal dimension of 1.7. Similar results were obtained for cellulose filter paper and glass filter paper. In the first the fractal-like kinetics is found at $4 \mathrm{~K}$ but not at $80 \mathrm{~K}$. This implies that most of the "fractal-like" aspects can be attributed to energetic rather than geometric disorder. The potential energy surface itself may include fractal-like canyons, etc., causing the reacting excitons to preferentially move in these fractal-like domains till they react. At low temperatures the energetic restrictions may be more important than the geometrical ones. Overall, at least qualitatively, the samples seem to obey the subordination law, ${ }^{(13)}$ according to which the "real" (geometrical) spectral dimension and the effective spectral dimension, due to energy disorder, simply multiply. (The Klafter et al. ${ }^{(13)}$ model for energy disorder is based on continuous times random walks.)

\subsection{Naphthalene Aggregates in Polymeric Glasses}

Samples of polymethylmethacrylate (PMMA) containing naphthalene up to $20 \%$ by weight show a mild fractal-like behavior at higher 


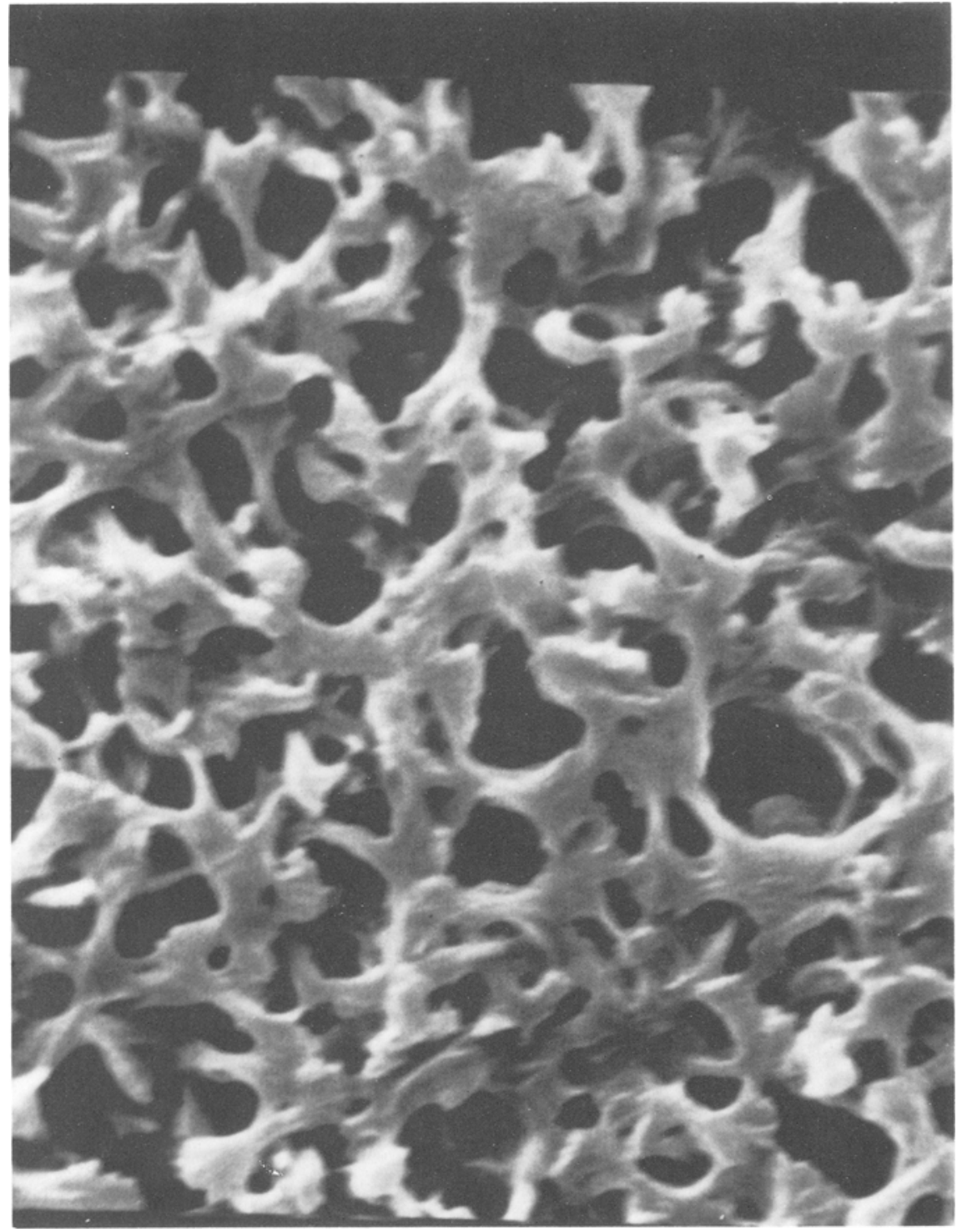

Fig. 5. Nylon membrane (Nyl 100, $0.2 \mu \mathrm{m}$ ), $7000 \times$ enlargement. 


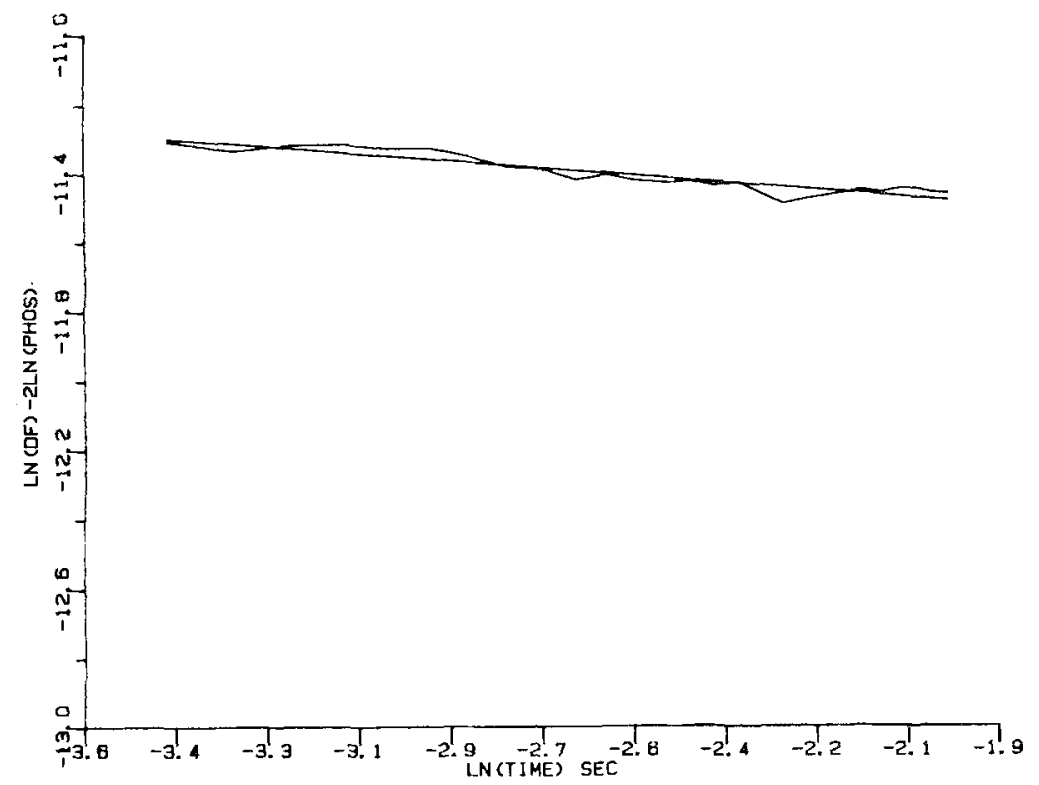

Fig. 6. Rate coefficient $k \equiv F / P^{2}$ vs. time on log-log plot for triplet exciton fusion (at $4 \mathrm{~K}$ ) on naphthalene embedded in nylon porous membrance (see Fig. 5). The fitted slope (straight line) gives $h=0.21$.

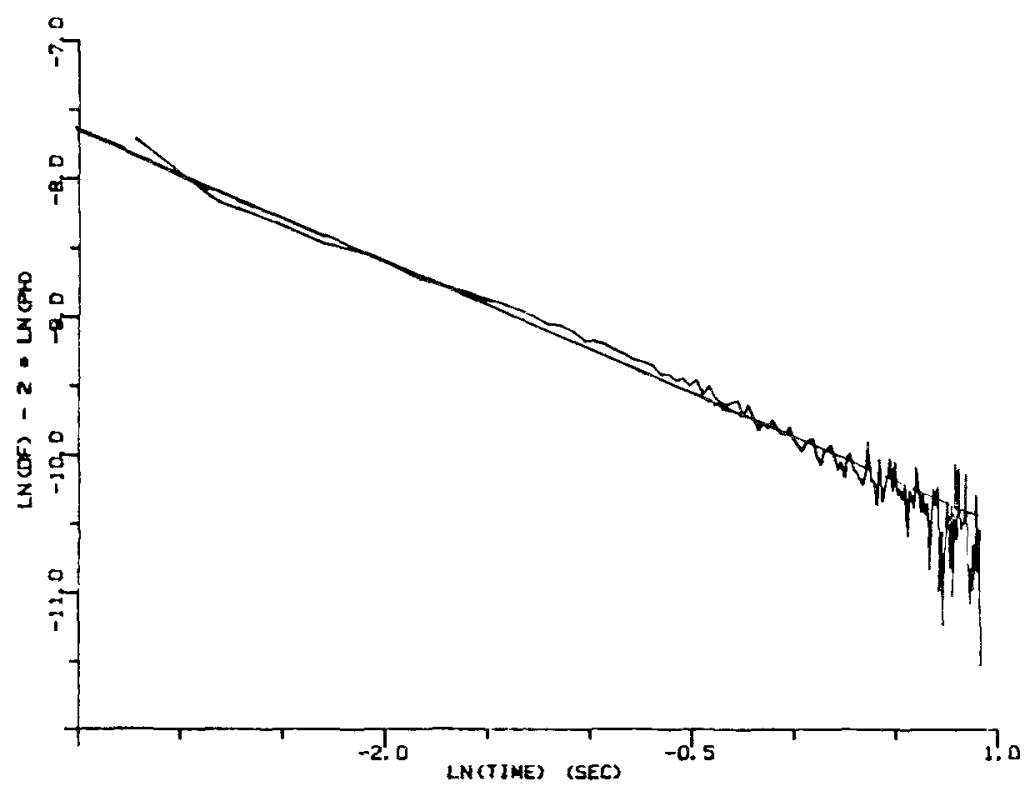

Fig. 7. Rate coefficient $k \equiv F / P^{2}$ vs. time on $\log -\log$ plot for tripled exciton fusion (at $6 \mathrm{~K}$ ) on naphthalene embedded in porous vycor glass $(0.003 \mu \mathrm{m})$. Fitted slope gives $h=0.44$. 
temperatures $^{(32)}(h=0.1-0.3)$. The relative roles of energetic and geometrical disorder are still under investigation for this doped (nonporous) "plexiglass."

\subsection{Naphthalene Films and Energetic Disorder}

These films were deposited at very low temperatures $(50 \mathrm{~K})$ from the vapor. The low-temperature samples $(6 \mathrm{~K})$ reveal clear-cut fractal-like kinetics $(h=0.45)$. It is obvious that the energetic disorder dominates the behavior of these samples. We believe that the fractal-like kinetics occurs in domain boundaries, which have lower excitation energies, as evidenced by spectroscopic studies, and thus really act as canyonlike energy funnels, first collecting the excitations from the semicrystalline domains (energy plateaus) and then channeling the excitations into these ramified (fractallike) domains toward reaction (annihilation). Again, the effective spectral dimension of this sample is about 1.1, similar to those of the porous glass and glass filter paper samples. Thus the question arises whether all naphthalene embedded porous membranes are more sensitive to the energetic disorder than to the geometrical shape. However, comparisons of various membranes, and especially those of glass filter paper to cellulose filter paper, reveal that there definitely is a geometric fractal component in the fractal-like kinetics of some of the samples. For instance, our tentative conclusion is that the pores of glass filter-paper indeed are fractal-like while those of cellulose filter-paper appear not to have a fractal geometry.

\section{SUMMARY}

A consistent picture of fractal-like chemical kinetics appears to emerge from the combination of scaling theory, Monte Carlo simulations, and exciton fusion reactions. The percolation cluster experiments, together with the relevant simulations, show the striking features of heterogeneous reactions in fractal-like geometries, with rate coefficients that decrease asymptotically with time and with effective reaction orders that can be skyhigh for a simple (elementary) binary reaction.

Energetic disorder, by itself or in tandem with geometric heterogeneities, leads to fractal-like effects that are hardly separable from purely geometric effects. In fact, the Scher-Montroll model ${ }^{(18)}$ of transport in disordered media is another representation of the same phenomenon. The mathematical waiting time distribution ("fractal time" ${ }^{(33)}$ ) is another way of describing a fractal-like potential energy surface.

Finally, one can use the fractal-like kinetics, exhibiting anomalous luminescence decays or equivalent features, as a diagnostic probe for spatial and/or energetic heterogeneity of the medium of interest. 


\section{NOTE ADDED IN PROOF}

Generalizations to steady-state $A+B$ reactions are under study.

\section{ACKNOWLEDGMENTS}

The simulations were performed by L. Anacker and J. Newhouse while the experiments were performed by L. Harmon, P. Klymko, S. Parus, and J. Prasad. Fruitful discussions with J. Klafter, R. Parson, and L. Sander are also acknowledged.

This research was supported in part by NIH Grant No. 2 R01 NS80116-16.

\section{REFERENCES}

1. S. Berry, S. A. Rice, and J. Ross, Physical Chemistry (Wiley, New York, 1980).

2. R. Kopelman, in Topics in Applied Physics, Vol. 15, F. K. Fong, ed. (Springer-Verlag, Berlin, 1976), p. 297.

3. M. V. Smoluchowski, Z. Phys. Chem. 92:129 (1917).

4. B. B. Mandelbrot, The Fractal Geometry of Nature (Freeman, San Francisco, 1983).

5. P. G. de Gennes, C. R. Acad. Sci. Ser. A 296:881 (1983), and references therein.

6. C. D. Mitescu and J. Rousseng, in Percolation Structures and Processes, G. Deutscher, R. Zallen, and J. Adler, eds. Ann. Israel Phys. Soc. 5:81 (1983), and references therein.

7. S. Chandrasekhar, Rev. Mod. Phys. 15:1 (1943).

8. R. M. Noyes, Prog. Reac. Kinetics 1:128 (1961).

9. N. G. van Kampen, Int. J. Quant. Chem. 16:101 (1982).

10. D. F. Calef and J. M. Deutch, Ann. Rev. Phys. Chem. 34:493 (1983).

11. P. Argyrakis and R. Kopelman, Chem. Phys. Lett. 61:187 (1979).

12. P. Argyrakis and R. Kopelman, Phys. Rev. B 22:1830 (1980).

13. J. Klafter, A. Blumen, and G. Zumofen, J. Stat. Phys. 36:561 (1984).

14. P. W. Klymko and R. Kopelman, J. Phys. Chem. 87:4565 (1983).

15. K. Kang and S. Redner, Phys. Rev. Lett. 52:955 (1984).

16. E. W. Montroll and G. H. Weiss, J. Math. Phys. (N.Y.) 6:167 (1965).

17. S. Alexander and R. Orbach, J. Phys. (Paris) Lett. 44:L13 (1982).

18. H. Scher and E. W. Montroll, Phys. Rev. B 12:2455 (1975).

19. P. Argyrakis, L. W. Anacker, and R. Kopelman, J. Stat. Phys. 36:579 (1984); L. W. Anacker et al., J. Stat. Phys. 36:591 (1984).

20. L. W. Anacker and R. Kopelman, J. Chem. Phys. 31:6402 (1984).

21. L. Sander (private communication, 1985).

22. F. Leyvraz and H. E. Stanley, Phys. Rev. Lett. 51:2048 (1983).

23. Y. Gefen, A. Aharony, and S. Alexander, Phys. Rev. Lett. 50:77 (1983).

24. J. S. Newhouse, P. Argyrakis, and R. Kopelman, Chem. Phys. Lett. 107:48 (1984).

25. J. S. Newhouse, Ph.D. thesis, The University of Michigan, Ann Arbor (1985).

26. L. W. Anacker, P. W. Klymko, and R. Kopelman, J. Lumin. 31/32:648 (1984).

27. P. W. Klymko, Ph.D. thesis, The University of Michigan, Ann Arbor (1984).

28. P. W. Klymko and R. Kopelman, J. Phys. Chem. 86:3686 (1982). 
29. P. Pfeifer, D. Avnir, and D. Farin, in Fractal Aspects of Materials: Metals and Catalyst Surfaces, Powders and Aggregates, B. B. Mandelbrot and D. E. Passoja, eds. (Materials Research Society, 1984), p. 4.

30. U. Even et al., Phys. Rev. Lett. 42:2164 (1984).

31. C. L. Yang, P. Evesque, and M. A. El-Sayed, J. Phys. Chem. 89:3442 (1985).

32. E. I. Newhouse and R. Kopelman, J. Lumin. 31/32:651 (1984).

33. M. F. Shlesinger, J. Stat. Phys. 36:639 (1984). 